\title{
Devising the guidelines: the techniques of uniportal video-assisted thoracic surgery-postoperative management and enhanced recovery after surgery
}

\author{
Luca Bertolaccini ${ }^{1}$, Alessandro Brunelli ${ }^{2}$ \\ ${ }^{1}$ Department of Thoracic Surgery, Maggiore Teaching Hospital, Bologna, Italy; ${ }^{2}$ Department of Thoracic Surgery, St. James's University Hospital, \\ Leeds, UK \\ Contributions: (I) Conception and design: All authors; (II) Administrative support: All authors; (III) Provision of study materials or patients: All \\ authors; (IV) Collection and assembly of data: All authors; (V) Data analysis and interpretation: All authors; (VI) Manuscript writing: All authors; (VII) \\ Final approval of manuscript: All authors. \\ Correspondence to: Luca Bertolaccini, MD, PhD, FCCP. Department of Thoracic Surgery, Maggiore Teaching Hospital, Largo Nigrisoli 2, 40133, \\ Bologna, Italy. Email: luca.bertolaccini@gmail.com.
}

\begin{abstract}
Kehlet first introduced the notion of enhanced recovery after surgery (ERAS). Moreover, in the last years, the fast-track programmes demonstrated a reduction of complications and the hospital length of stay in general surgery. ERAS involves a multidisciplinary to development the value of care introducing the evidence-based knowledge into practice. ERAS has spread to other surgical specialities, showing the same improvements regarding clinical outcomes and costs. Therefore, there are numerous guidelines official published by the ERAS Society for many specialities, and many meta-analyses recognised the benefits of ERAS. ERAS pathways have demonstrable advantages in some specialities such as colorectal surgery. There is emerging evidence of ERAS efficacy in thoracic surgery. ERAS is safe and not increase postoperative morbidities, and ERAS guidelines should encourage future researches to address current knowledge gaps. Nevertheless, further prospective and randomised studies on the ERAS protocol, including the ones based on the uniportal video-assisted thoracic surgery (UniVATS), and focussing more on longitudinal outcomes over costs will be necessary. In fact, in the era of minimally invasive surgery traditional findings may not be appropriate to capture all benefits provided by ERAS. There is, therefore, a need to switch focus to endpoints linked to value in health care and patient centred efficiency.
\end{abstract}

Keywords: Enhanced recovery after surgery (ERAS); lung cancer; uniportal video-assisted thoracic surgery (UniVATS)

Submitted Nov 27, 2018. Accepted for publication Jan 15, 2019.

doi: $10.21037 /$ jtd.2019.01.62

View this article at: http://dx.doi.org/10.21037/jtd.2019.01.62

\section{Introduction}

Kehlet first introduced the notion of enhanced recovery after surgery (ERAS) (1). Moreover, in the last years, the fast-track programmes demonstrated a reduction of complications and the hospital length of stay in general surgery. ERAS involves a multidisciplinary team to develop the quality of care introducing the evidence-based knowledge into clinical practice (2). ERAS has spread to other surgical specialities, showing the same improvements regarding clinical outcomes and costs (3). Therefore, there is some guidelines official published by the ERAS Society for many specialities, and numerous meta-analyses recognised the benefits of ERAS (2).

In this paper, we have discussed the role of ERAS in thoracic surgery and on the possible compliance of uniportal video-assisted thoracic surgery (UniVATS) for the ERAS protocol. 


\section{ERAS protocols in thoracic surgery}

Cerfolio et al. realised a fast-track program for open lobectomies with a specific focus on preoperative patient tutoring, epidural usage, standardised aggressive removal of catheters and drains, postoperatively early mobilisation and a daily strategy with predictable discharge day on the fourth postoperative day. These interventions enabled early discharge without unfavourably upsetting mortality or morbidity (4). Nevertheless, although the individual elements of enhanced recovery are well known and applied in isolation since decades in our specialities (i.e., pain control, fluid restriction, early mobilisation, etc.) the adoption of the ERAS as an integrated multimodal package is relatively recent in thoracic surgery (5). Several independent factors emerged as influent for ERAS as early mobilisation and preoperative carbohydrate drinks. On the other hand, intentional admission to the intensive therapy unit is an institutional issue related to the delayed discharge, although it does not affect morbidity. Scientific evidence suggested, in thoracic surgery, ERAS paths as superior to conventional care, and the protocol compliance improves clinical outcomes (3).

In thoracic surgery, many of the principles of enhanced recovery are adjusted to ERAS protocols. The prevention of postoperative complications is associated with reduced hospitalisation, early recovery, decreased healthcare costs, and good postoperative quality of life. ERAS protocol presents as in general surgery, an evidence-based approach to patient care. Even in thoracic surgery, ERAS consists of three phases: preoperative, intraoperative, and postoperative (3).

The use of a minimally invasive approach is the mainstay of ERAS in any speciality. In our field, the use of VATS as opposed to thoracotomy has shown to probably be the most critical element contributing the most to the positive effect of ERAS and likely obscuring the influence of other factors. This is probably the main explanation of the lack of benefit observed in a pre-ERAS versus post ERAS comparative study focussed on VATS lobectomies only (6). Also, other studies have shown that the benefits of ERAS are more evident after thoracotomy compared to VATS lung resections $(7,8)$.

Indeed, VATS facilitate the delivery and application of other elements of ERAS such as early mobilisation. Early mobilisation is known as a protecting factor for reducing complications, while a recent systematic review did not recognise any benefits (due to the poor methodological quality of studies) (9). Also, in VATS, targeted postoperative nausea and vomiting control, avoidance of epidural analgesia, a standardised analgesia treatment without opiates (if possible), and avoidance of fluid excess are typical and more easily achieved. A digital chest drainage system with aggressive management also helps the early recovery (5).

Therefore, positive features of perioperative care combined into ERAS procedures are more critical in open processes versus VATS. In general, it is essential to comprehend the likely benefit of applying additional elements of ERAS when many fundamental elements are now in place, mainly when determining if it is worth the necessary cost and resources (10).

A multidisciplinary approach is essential, and many mechanisms of ERAS path are out of the surgeon's straight control. Consequently, collaboration and buy-in from crucial workers are critical for productive employment of an ERAS procedure. There are also numerous reasonable mechanisms that are likely to progress patient compliance and the overall perioperative involvement (10).

The recent European Society of Thoracic Surgeons (ESTS) guidelines outline recommendations for the perioperative management of patients undergoing thoracic surgery. Some suggestions are broad or founded on information generalised from other specialities (alcohol abuse management, preoperative anaemia management, carbohydrate treatment, venous thromboembolism prophylaxis and early enteral feeding). Recommendations are based on the quality of the evidence and steadiness between desirable and undesirable effects. Since the methodological proposal for most of the ERAS statement is active, the usage of a systematic ERAS pathway could improve the outcomes (11).

\section{ERAS in UniVATS}

Since its introduction, UniVATS has emerged as a feasible alternative to multiport VATS approach to patients with non-small cell lung cancer. Therefore, thoracic surgeons have been able to perform increasingly complex thoracic procedures and have included this approach in their surgical armamentarium $(12,13)$. UniVATS lobectomy did not rise postoperative morbidity, demonstrating that it was safe and practicable, yielded enhanced postoperative recovery (14). The amalgamation of the smallest minimally invasive technique with the well-established ERAS path could lead to an optimised hospital stay and treatment, particularly lung cancer patients (15). Therefore, it is worthwhile to set up ERAS for UniVATS. Since the 
incision of UniVATS is limited to one intercostal space and the length of incisions are less than those in multiport VATS, the trauma to blood vessels, muscles, and nerves should be reduced, with lesser postoperative discomfort and chest paraesthesia. Also, the hospital length of stay due to the reduced trauma and the extensions of indications for drainage removal to $<400 \mathrm{~mL} / 24$ hours should be shorter in UniVATS (14). The combination between UniVATS and ERAS may offer true and essential benefits for the patient regarding recovery, reduction of postoperative morbidity, and improvement of the quality of life. Undeniably, a growing number of papers have already defined the efficiency of the ERAS protocols regarding economic benefits and recovery for the patients both for other surgical procedures and UniVATS. Nevertheless, only a low number of reports, assess the combined results (15). The application of ERAS could contribute to improving the quality of care, patient safety, and team efficiency and ultimately could save money. Nevertheless, traditional endpoints (e.g., length of stay and early outcomes) are not able to capture the benefits of ERAS entirely. VATS is arguably the most crucial element of an ERAS protocol, likely blurring the effects of other components (16).

\section{Conclusions}

ERAS pathways have demonstrable advantages in some specialities such as colorectal surgery. There is emerging evidence of ERAS efficacy in thoracic surgery. ERAS is safe and not increase postoperative morbidities, and ERAS guidelines should encourage future researches to address current knowledge gaps. Nevertheless, further prospective and randomised studies on the ERAS protocol, including the ones based on the UniVATS, and focussing more on longitudinal outcomes over costs will be necessary. In fact, in the era of minimally invasive surgery traditional findings may not be appropriate to capture all benefits provided by ERAS. There is, therefore, a need to switch focus to endpoints linked to value in health care and patient centred efficiency (16).

\section{Acknowledgments}

None.

\section{Footnote}

Conflicts of Interest: The authors have no conflicts of interest to declare.

\section{References}

1. Kehlet H. ERAS Implementation-Time To Move Forward. Ann Surg 2018;267:998-9.

2. Bertolaccini L, Rocco G, Crisci R, et al. Enhanced recovery after surgery protocols in video-assisted thoracic surgery lobectomies: the best is yet still to come? J Thorac Dis 2018;10:S493-6.

3. Dinic VD, Stojanovic MD, Markovic D, et al. Enhanced Recovery in Thoracic Surgery: A Review. Front Med (Lausanne) 2018;5:14.

4. Cerfolio RJ, Pickens A, Bass C, et al. Fast-tracking pulmonary resections. J Thorac Cardiovasc Surg 2001;122:318-24.

5. Rogers LJ, Bleetman D, Messenger DE, et al. The impact of enhanced recovery after surgery (ERAS) protocol compliance on morbidity from resection for primary lung cancer. J Thorac Cardiovasc Surg 2018;155:1843-52.

6. Brunelli A, Thomas C, Dinesh P, et al. Enhanced recovery pathway versus standard care in patients undergoing videoassisted thoracoscopic lobectomy. J Thorac Cardiovasc Surg 2017;154:2084-90.

7. Martin LW, Sarosiek BM, Harrison MA, et al. Implementing a Thoracic Enhanced Recovery Program: Lessons Learned in the First Year. Ann Thorac Surg 2018;105:1597-604.

8. Van Haren RM, Mehran RJ, Mena GE, et al. Enhanced Recovery Decreases Pulmonary and Cardiac Complications After Thoracotomy for Lung Cancer. Ann Thorac Surg 2018;106:272-9.

9. Castelino T, Fiore JF, Jr., Niculiseanu P, et al. The effect of early mobilization protocols on postoperative outcomes following abdominal and thoracic surgery: A systematic review. Surgery 2016;159:991-1003.

10. Semenkovich TR, Hudson JL, Subramanian M, et al. Enhanced Recovery After Surgery (ERAS) in Thoracic Surgery. Semin Thorac Cardiovasc Surg 2018;30:342-9.

11. Batchelor TJP, Rasburn NJ, Abdelnour-Berchtold E, et al. Guidelines for enhanced recovery after lung surgery: recommendations of the Enhanced Recovery After Surgery (ERAS(R)) Society and the European Society of Thoracic Surgeons (ESTS). Eur J Cardiothorac Surg 2019;55:91-115.

12. Rocco G. One-port (uniportal) video-assisted thoracic surgical resections--a clear advance. J Thorac Cardiovasc Surg 2012;144:S27-31. 
13. Rocco R, Rocco G. Future study direction on single port (uniportal) VATS. J Thorac Dis 2016;8:S328-32.

14. Huang H, Ma H, Chen S. Enhanced recovery after surgery using uniportal video-assisted thoracic surgery for lung cancer: A preliminary study. Thorac Cancer 2018;9:83-7.

15. Ismail M, Swierzy M, Nachira D, et al. Fast-Tracking Patients Through the Diagnostic and Therapeutic

Cite this article as: Bertolaccini L, Brunelli A. Devising the guidelines: the techniques of uniportal video-assisted thoracic surgery-postoperative management and enhanced recovery after surgery. J Thorac Dis 2019;11(Suppl 16):S2069-S2072. doi: 10.21037/jtd.2019.01.62
Pathways of Intrathoracic Conditions: The Role of Uniportal Video-Assisted Thoracic Surgery. Thorac Surg Clin 2017;27:425-30.

16. Brunelli A. Enhanced recovery pathways in thoracic surgery: Time for a version 2.0. J Thorac Cardiovasc Surg 2018;155:2758-9. 